\title{
Clinical Analysis of Subarachnoid Haemorrhage of Unknown Aetiology (SAHUE)
}

\author{
R. Srinivas ${ }^{1}$, Mohammed Naleer ${ }^{2}$, Anupama Guttimdeevi ${ }^{3}$ \\ 1Department of Neurosurgery, Sri Ramachandra Institute of Higher Education and Research, \\ Chennai, Tamilnadu, India. ${ }^{2}$ Department of Neurosurgery, Sri Ramachandra Institute of Higher \\ Education and Research, Chennai, Tamilnadu, India. ${ }^{3}$ Tambaram East, Chennai, Tamilnadu, India.
}

\section{ABSTRACT}

\section{BACKGROUND}

Spontaneous subarachnoid haemorrhage accounts for 15 cases per lakh population. There are few reports of patients with perimesencephalic haemorrhage and few reports with limited number of patients. Therefore, the aim of the present study was to investigate the clinical course and outcome in patients with SAH.

\section{METHODS}

In our study, we identified a total of 55 patients admitted to the Neurosurgery department of Sri Ramachandra Institute of Higher Study and Research with subarachnoid haemorrhage between 2018 and 2019. Medical records of all patients who underwent treatment for subarachnoid haemorrhage from July 2018 to April 2019 in the tertiary-care center were reviewed from a prospectively collected database. A detailed analysis was performed on potential predictors of postoperative complications, including age, gender, and type of admission.

\section{RESULTS}

Negative sub-arachnoid haemorrhage angiogram exhibited very mild prognosis than with aneurysmal sub-arachnoid haemorrhage. All patients had presence of blood either in perimesencephalic cisterns and in the lateral cisterns. The prognosis of patient varies based on the thickness of hematoma and people with hydrocephalus had poor prognosis.

\section{CONCLUSIONS}

Each patient with subarachnoid haemorrhage should be monitored as an individual case and to prevent death it is important to identify patients to reduce the aneurysms and modify the risk factors associated with.

\section{KEY WORDS}

Spontaneous Subarachnoid Haemorrhage, Clinical Outcome, Radiological Evaluation and Prediction.
Corresponding Author:

Dr. R. Srinivas,

M. Ch. Neurosurgery,

Assistant Professor

SRIHER, Porur, Tamilnadu,

Chennai-600116, India.

E-mail: drsrinivas74@gmail.com

DOI: $10.14260 / j e m d s / 2020 / 435$

How to Cite This Article:

Srinivas R, Naleer M, Guttimdeevi A. Clinical analysis of subarachnoid haemorrhage of unknown aetiology (Sahue). J. Evolution Med. Dent. Sci. 2020;9(28):1997-2001, DOI: 10.14260/jemds/2020/435

Submission 10-04-2020,

Peer Review 04-06-2020,

Acceptance 10-06-2020,

Published 13-07-2020.

Copyright (c) 2020 JEMDS. This is an open access article distributed under Creative Commons Attribution License [Attribution 4.0 International (CC BY 4.0)] 


\section{BACKGROUND}

Spontaneous subarachnoid haemorrhage (SAH) accounts for 15 per lakh population. In 1985, SAH was identified as the pattern of haemorrhage anterior to the midbrain without intraparenchymal or intra ventricular extension without any bleeding on DSA and a benign clinical condition. SAH is usually caused by the rupture of an intracranial aneurysm. But only 13 $\%$ was found to be negative for any intracranial aneurysms. Such patients with angiography negative SAH have lower risk of rebleeding and their blood distribution is termed as perimesencephalic or Prepontine.(1,2) Perimesencephalic haemorrhage is a subset of subarachnoid haemorrhage. It has been identified in recent years that patients with an aneurysmal subarachnoid haemorrhage have a reduced life expectancy. Spontaneous subarachnoid haemorrhage occurs by sudden onset headache which is usually described as the worst ever headache described by the patient. The patient presents with neck pain along with headache and usually it may be associated with vomiting also. The patient also has loss of consciousness based on the severity of haemorrhage. $(3,4)$ Based on the associated any intra-parenchymal haemorrhage there may even be neurological deficits associated with blurring of vision. Bleeding cannot be demonstrated in the spontaneous SAH patients. Patients with peri-mesencephalic SAH are believed to attain a good outcome and they are found to have a lower risk of rebleeding.(1,3) Scribed as the worst ever headache described by the patient. The patient presents with neck pain along with headache. Usually it may be associated with vomiting also. The patient also has loss of consciousness based on the severity of haemorrhage. Based on the associated any intraparenchymal haemorrhage there may even be neurological deficits associated with blurring of vision.

Haemorrhagic origin is unidentifiable in $10 \%-20 \%$ of patients presenting with spontaneous subarachnoid haemorrhage (SAH). While the patients in such cases do well clinically, there is a lack of long-term angiographic follow-up. Hydrocephalus and delayed cerebral ischemia, while infrequent, do occur in SAH of unknown origin. Long-term neurological outcomes are generally good. A thorough evaluation to rule out an aetiology of haemorrhage is necessary; however, imaging beyond 6 weeks from ictus has little utility, and rebleeding is unexpected. There has been a worldwide shift towards ambulatory surgeries and a growing body of evidence has surfaced in the past decade showing a predilection towards outpatient thyroid surgery, with an emphasis on differentiated selection criteria.(1) This is more evident in the United States, where ambulatory procedures account for more than $70 \%$ of all elective surgical procedures.(5) This shift is partly driven by the advantage of healthcare cost containment, especially in times where costs may affect healthcare access and quality of care. Additionally, short-stay procedures contribute to an increase in hospital bed availability and a decrease in the incidence of postoperative nosocomial infections. Sun et al. reported that the total number of outpatient thyroid surgeries increased by $61 \%,{ }^{(6)}$ which reaffirms the advantages surgeons see in an ambulatory setting. Multiple institutions have adopted a practice of carrying out these surgeries on a short-stay basis, where the patient is held only for a short observation period after surgery.(1) Despite the conflicting opinions about the feasibility of short-stay thyroidectomy, multiple reports have demonstrated that it is safe, provided that cases are selected properly and a systematic perioperative care protocol is followed.(1,2,7) There are few reports on the patients with perimesencephalic haemorrhage and few reports with limited number of patients. Therefore, the aim of the present study was to investigate the clinical course and outcome in patients with SAH.

\section{METHODS}

\section{Ethical Consideration}

The study was conducted after the approval from the institutional ethics committee. Participants were explained about the purpose and informed written consent was obtained from them before conducting the study. Confidentiality of the responses were assured and maintained throughout the study.

\section{Research Design and Approach}

Quantitative research approach was used to assess the Clinical Analysis of Subarachnoid Haemorrhage of Unknown Origin (SAHUE) at Sri Ramachandra Hospital in Chennai. Purposive sampling technique was used to select samples. During the study period, 55 samples were selected based on the inclusion and exclusion criteria.

\section{Setting}

Chennai, is metro city in south of India surrounded by many medical education institution, it serves more than 5000 patients per day. Its covers a population of 20000000 inhabitants according to 2011 National Census. The population is served by over 15 regional hospitals and 8-10 university teaching hospitals including Sri Ramchandra Institute of Higher Education and Research Hospital. Medical care is at charge of the patients who have a limited access to hospitals, high-quality medical services, and diagnostic technologies, including CT (computerized tomography) and MRI (Magnetic Resonance Imaging). This University accounts Emergency Department, Resuscitation Department, Neurology Department, and Neurosurgery Department which admitted patients with subarachnoid haemorrhage. All the patients with SAH were admitted to Emergency Department and after were transferred to Neurology Department or Resuscitation Department. Only patients with aneurysmal SAH were admitted to Neurosurgery Department.

In our study, we identified a total of 55 patients admitted to the Neurosurgery department of Sri Ramachandra institute of higher study and Research with subarachnoid haemorrhage between 2018 and 2019. Subarachnoid haemorrhage was confirmed by radiologically via urgent computed tomography (CT) scan without contrast. Traditionally, a negative CT scan is followed with lumbar puncture. However, non-contrast CT followed by CT angiography (CTA) of the brain can rule out SAH with greater than $99 \%$ sensitivity. The patients were subjected to four-vessel 3D digital subtraction angiography (DSA) within 24 hours of admission and repeat DSA done at an interval of 2 weeks. IV 3D DSA was obtained with rotational angiographies which was performed on a C-Arm (Infinix Celeve VS; Toshiba, Tokyo, Japan). This covers a total angular 
range of $200^{\circ}$, with a first rotation of $40^{\circ} / \mathrm{s}$ to acquire the mask images, a second rotation to return to the starting position, and a third rotation of $40 \% \mathrm{~s}$ to acquire the opacified images. The type of detector in this system was an image intensifier (RTP12303J-G9E; Toshiba). An 18-gauge angiocatheter was inserted into the right antecubital vein and linked with a connective tube for power injection. First, $15 \mathrm{~mL}$ of nonionic contrast medium (Omnipaque 350; Daiichi Seiyaku, Tokyo, Japan) was injected with a pump at a rate of $9 \mathrm{~mL} / \mathrm{s}$. The delay time from the start of contrast injection to the time when the common carotid arteries were filled with contrast was measured with DSA. Then $50 \mathrm{~mL}$ of the contrast medium was injected at a rate of $9 \mathrm{~mL} / \mathrm{s}$. The acquisition of source images was started after the start of contrast injection. The acquisition matrix was $512 \times 512$. This protocol resulted in a rotational series of 200 subtracted images. Rotational angiography data were automatically transferred to a personal computer (XIDF100A; Toshiba), which reconstructed 3D volume data. The matrix of transferred data were transformed from $512 \times 512$ to $256 \times 256$ by voxel addition. The 3D volume data were transferred to a commercially available multimodality 3D workstation (ZIO M900TXA; ZIO Software, Tokyo, Japan), processed, and presented. Available visualization of algorithms included maximum intensity projection (MIP) and volume rendering. A computed tomography scan was performed within 72 hours after the onset of headache showing a perimesencephalic pattern of haemorrhage. The initial CT scan of the patient's brain revealed blood either in peri-mesencephalic cisterns which was called as localized blood and people who had blood in both peri-mesencephalic cisterns and in the lateral cisterns were classified as diffuse collection of blood radiologically. The absence of a saccular aneurysm on computed tomographic angiography or conventional angiography was noted. The patients who had initial angiogram negative finding for aneurysm were taken into study.

\section{The Glasgow Coma Scale (GCS)}

It is a neurological scale which aims to give a reliable and objective way of recording the state of a person's consciousness for initial as well as subsequent assessment. A person is assessed against the criteria of the scale, and the resulting points give a person's score between 3 (indicating deep unconsciousness) and either 14 (original scale) or 15 (more widely used, modified or revised scale). GCS was used to assess a person's level of consciousness after a head injury, and the scale is widely used by emergency medical services, nurses, and physicians as being applicable to all acute medical and trauma patients. In these hospitals, it is also used in monitoring patients in intensive care units. The score is expressed in the form "GCS 9= E2 V4 M3 at 07:35". Generally, brain injury is classified as: Severe, GCS $<8-9$; Moderate, GCS 8 or $9-12$ (controversial); Minor, GCS $\geq 13$.

\section{Statistical Analysis}

The collected data were grouped and analyzed based on percentage, mean and standard deviation.
RESULTS

Totally, we identified 55 cases of spontaneous subarachnoid haemorrhage during the study period. All the cases of subarachnoid haemorrhage were identified by CT and clinical evaluation of the patients. The mean age of the 55 patients was 27.5 \pm 1.3 (25 men and 30 women) (Table 1). 47 patients had Glasgow Coma Scale (GCS) between 13-14 both after admission and on discharge. Original CT films that were used to measure the volume of subarachnoid, intraventricular, and intraparenchymal haemorrhage were available for all the 55 patients included in this study. The patient results have been tabulated below and the factors studied have been analysed. Results of radiology of haematoma showed that majority of them had local haematoma, and other major had hypertension as reason of haematoma.

\begin{tabular}{|ccc|}
\hline $\begin{array}{c}\text { Characteristics Studied } \\
\text { 1. Sex }\end{array}$ Number of Patients & Percentage of Cases \\
Male & 25 & $45 \%$ \\
Female & 30 & $55 \%$ \\
2. Glasgow Coma Scale (GCS) on Admission & \\
$13-14$ & 47 & $85 \%$ \\
$3-4$ & 8 & $15 \%$ \\
3. Hypertension & 47 & \\
Yes & 8 & $85 \%$ \\
No & 4. Radiology Haematoma & $15 \%$ \\
Diffuse & 8 & $15 \%$ \\
Local & 47 & $85 \%$ \\
Diabetes & 12 & $22 \%$ \\
Hypertension & 13 & $23 \%$ \\
Anticoagulants & 3 & $5 \%$ \\
5. Glasgow Coma Scale (GCS) on Discharge & \\
14 & 47 & $85 \%$ \\
8 & 8 & $15 \%$ \\
6. Repeat DSA & 7 & $14.30 \%$ \\
Positive & 48 & $84.70 \%$ \\
Negative & Table 1. Characteristics of Patients Studied \\
\multicolumn{2}{r}{ and the Factors Analysed } \\
\hline
\end{tabular}

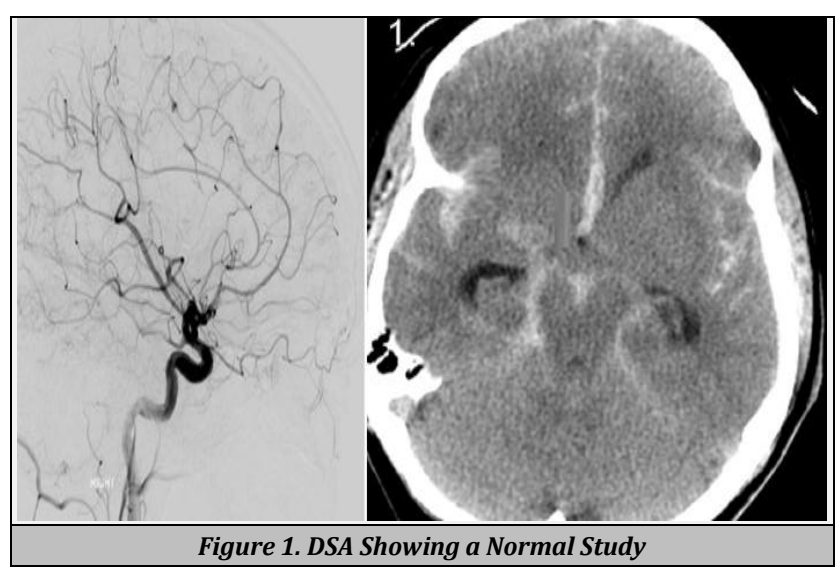

The image below shows the preliminary illustration of a patient with subarachnoid haemorrhage and a normal DSA (Figure 2 \& 3). Non-contrast head CT examples of SAH. Perimesencephalic SAH: axial image from a non-contrast head CT demonstrates acute $\mathrm{SAH}$ in the prepontine and interpeduncular cistern, consistent with a perimesencephalic pattern of SAH (A). Sulcal SAH: axial image from a non-contrast head CT demonstrates acute SAH in the left precentral sulcus and in the sulci overlying the left middle frontal gyrus, consistent with a sulcal pattern of SAH (B). Diffuse SAH: axial image from a non-contrast head CT demonstrates acute SAH in the bilateral Sylvian fissures, overlying the sulci of the bilateral temporal lobes, consistent with a diffuse pattern of SAH. Note 
also intraventricular haemorrhage within the third ventricle (C). Isolated IVH: axial image from a non-contrast head CT demonstrates acute intraventricular haemorrhage casting the right lateral ventricle (D).

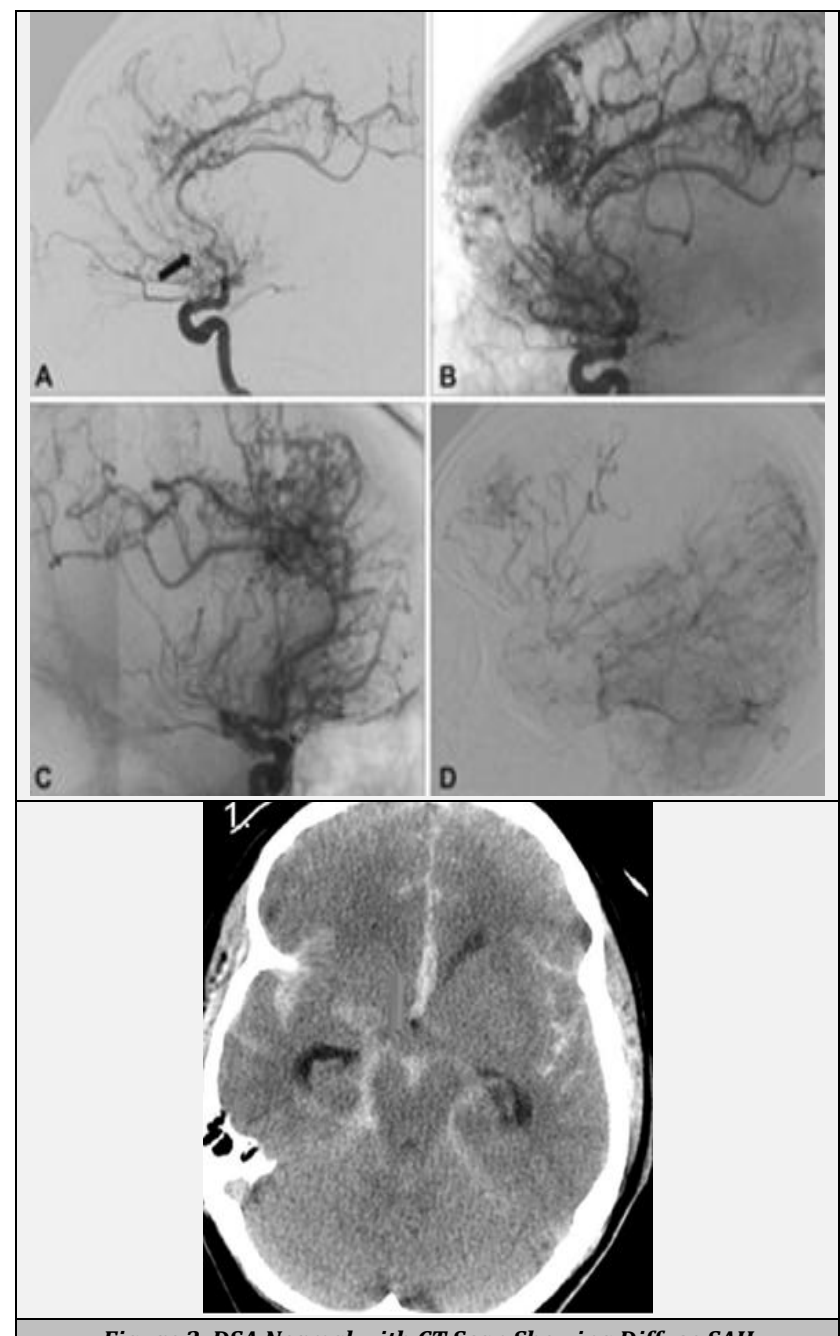

Figure 2. DSA Normal with CT Scan Showing Diffuse SAH

\section{DISCUSSION}

A perimesencephalic haemorrhagic pattern is actually due to the rupture of a posterior circulation aneurysm. $(5,6)$ Thus, the viewing of the intracranial vessels becomes mandatory. The use of CTA as a primary imaging and triage technique in patients presenting with SAH results in decreased time to diagnosis and reduced medical costs. At our tertiary care center, all patients presenting with SAH undergo a CTA of the head, and this technique identified an aneurysm as the cause of SAH. For much of this period, the bulk of patients who underwent subsequent craniotomy for ruptured aneurysms did not undergo preoperative DSA examination.In our study, population with perimesencephalic haemorrhage there was no mortality encountered among the patients in comparison to the general population and the patients were recovered with full independence to day to day activities. Reports suggested that negative sub-arachnoid haemorrhage angiogram exhibited very mild prognosis than with aneurysmal sub-arachnoid haemorrhage. $(7,8)$ Many patients experience morbidity and mortality due to haemorrhage. For such cases repeat DSA is performed to identify the source of bleeding but the sources still remain undetected.(9) In our study, we included patients with only negative aneurysm. We studied perimesencephalic haemorrhage disease due to causes other than intracranial aneurysm. The thicker the blood there was more deficits found and recovery delay was found. Theoretically when no aneurysm was found they say it is of venous origin which is still doubtful because no evidence found till now. But DSA done after 4 weeks interval revealed positive aneurysm in 14 percent of patients. Thus, our study corroborates the characteristics of aneurysm which does not occur as one-time life effect but occurs in all the patients who had survived an aneurysmal subarachnoid haemorrhage episode. the patients in this study underwent a DSA within 24 hours of admission and they had one more DSA done at an interval of 2 weeks. The patients who had initial angiogram negative finding for aneurysm were taken into study. All the patients had an initial CT scan of brain which revealed blood either in perimesencephalic cisterns which was called as localised blood and people who had blood in both perimesencephalic cisterns and in the lateral cisterns were classified as diffuse collection of blood radiologically. The thicker the blood there was more deficits found and recovery delay was found. Theoretically when no aneurysm was found they say it is of venous origin which is still doubtful because no evidence found till now

We could not have some reliable data on the non-specific symptoms of the patients such as headache, dizziness, fatigue etc., and hence we could not study these in detail. Only computed tomography and DSA was performed in our study to rule out the negative aneurysm. Some previous studies state that this may pose a risk of missing out an aneurysm. But in contrary few previous reports suggested that computed tomographic angiography alone is the best diagnostic strategy to rule out any aneurysm. Our study also confirmed the same with hardly any negative prediction of computed tomographic angiography for an aneurysm in the patients with a perimesencephalic haemorrhage. Five per cent of patients in our study were under anticoagulant therapy but the adequate risk of anticoagulant therapy on patients with peri-mesencephalic haemorrhage is not available.

The prognosis of patient varies based on the thickness of hematoma and people with hydrocephalus had poor prognosis.(10) Hydrocephalus developed in 7 of your patients for which intervention in the form of EVD was done in 4 patients and 2 patients underwent permanent V.P. shunt. Blood in localized basal cisterns had a good prognosis than patients with diffuse blood in all the cisterns. Each patient with subarachnoid haemorrhage should be monitored and treated since the mortality of patients with subarachnoid haemorrhage may not be reduced due to any therapy. The only way to prevent death is to safely identify patients to reduce the aneurysms and modify the risk factors associated with.

\section{CONCLUSIONS}

A perimesencephalic haemorrhagic pattern is due to posterior circulation aneurysm rupture, which becomes mandatory to visualise. Study suggested that negative sub-arachnoid haemorrhage angiogram exhibited very mild prognosis than 
with aneurysmal sub-arachnoid haemorrhage. Here, We studied perimesencephalic haemorrhage disease due to causes other than intracranial aneurysm., which showed that all the patients had presence of blood either in perimesencephalic cisterns and in the lateral cisterns. The prognosis of patient varies based on the thickness of hematoma and people with hydrocephalus had poor prognosis. This study concludes that each patient with subarachnoid haemorrhage should be monitored as an individual case and to prevent death it is important to identify patients to reduce the aneurysms and modify the risk factors associated with.

\section{REFERENCES}

[1] Broderick JP, Brott TG, Duldner JE, et al. Initial and recurrent bleeding are the major causes of death following subarachnoid haemorrhage. Stroke 1994;25(7):1342-7.

[2] McMahon J, Dorsch N. Subarachnoid haemorrhage of unknown aetiology: what next? Crit Rev Neurosurg 1999;9(3):147-55.

[3] Ildan F, Tuna M, Erman T, et al. Prognosis and prognostic factors in nonaneurysmal perimesencephalic haemorrhage: a follow-up study in 29 patients. Surg Neurol 2002;57(3):160-5.
[4] van Gijn J, van Dongen KJ, Vermeulen $M$, et al. Perimesencephalic haemorrhage: a nonaneurysmal and benign form of subarachnoid haemorrhage. Neurology 1985;35(4):493-7.

[5] Canhao P, Ferro JM, Pinto AN, et al. Perimesencephalic and nonperimesencephalic subarachnoid haemorrhages with negative angiograms. Acta Neurochir (Wien) 1995;132(1-3):14-9.

[6] Garcia-Rodriguez LA, Gaist D, Morton J, et al. Antithrombotic drugs and risk of haemorrhagic stroke in the general population. Neurology 2013;81(6):566-74.

[7] van der Worp HB, Fonville S, Ramos LMP, et al. Recurrent perimesencephalic subarachnoid haemorrhage during antithrombotic therapy. Neurocrit Care 2009;10(2):20912.

[8] Konczalla J, Platz J, Schuss P, et al. Non-aneurysmal nontraumatic subarachnoid haemorrhage: patient characteristics, clinical outcome and prognostic factors based on a single-center experience in 125 patients. BMC Neurology 2014;14:140.

[9] Hawkins TD, Sims C, Hanka R. Subarachnoid haemorrhage of unknown cause: a long term follow-up. J Neurol Neurosurg Psychiatry 1989;52(2):230-5.

[10] Almandoz JED, Jagadeesan BD, Refai D, et al. Diagnostic yield of repeat catheter angiography in patients with catheter and computed tomography angiography negative subarachnoid haemorrhage. Neurosurgery 2012;70(5):1135-42. 